(C) 2015 the authors. Access to this work was provided by the University of Maryland, Baltimore County (UMBC) ScholarWorks@UMBC digital repository on the Maryland Shared Open Access (MD-SOAR) platform.

Please provide feedback

Please support the ScholarWorks@UMBC repository by emailing scholarworks-group@umbc.edu and telling us what having access to this work means to you and why it's important to you. Thank you. 


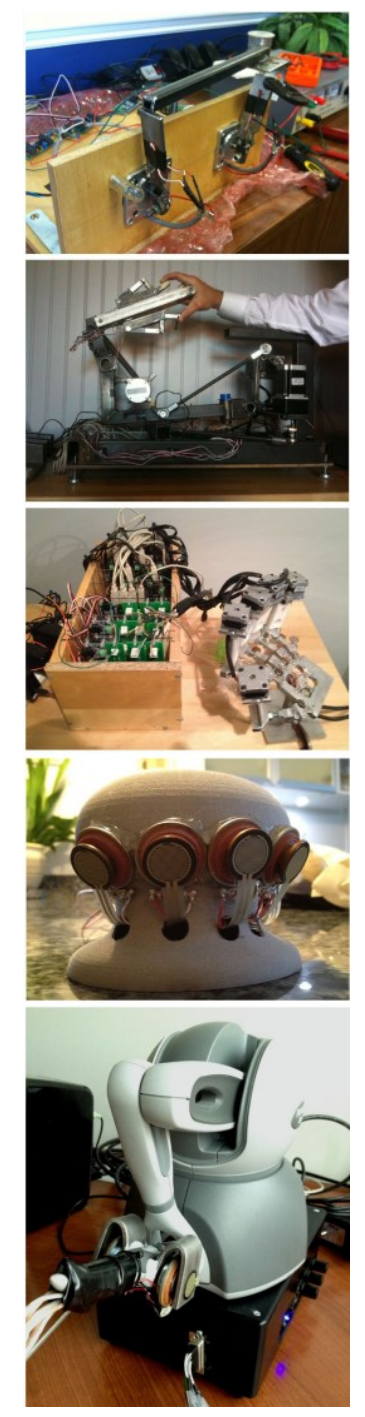

Figure 1: Design evolution

\section{Tangibly Enhancing Haptics}

\section{Philip Feldman}

UMBC

1000 Hilltop Circle

Baltimore, MD 21250 USA

feld1@umbc.edu

\section{Ravi Kuber}

UMBC

1000 Hilltop Circle

Baltimore, MD 21250 USA

rkuber@umbc.edu
Permission to make digital or hard copies of part or all of this work for personal or classroom use is granted without fee provided that copies are not made or distributed for profit or commercial advantage and that copies bear this notice and the full citation on the first page. Copyrights for third-party components of this work must be honored. For all other uses, contact the owner/author(s). Copyright is held by the author/owner(s).

TEI 2015, January 15-19, 2015, Stanford, CA, USA

ACM 978-1-4503-3305-4/15/01

http://dx.doi.org/10.1145/2677199.2687903

\begin{abstract}
In this paper, we describe the development of a novel, low-cost, compact sensor/actuator that can be

integrated with a commercially-available haptic device, such as the Geomagic Touch, to provide additional capability when grasping virtual objects. The focus of this paper is to investigate the impact of modality on a 'picking and placing' task using the prototype. The results of this study suggest that fewer errors were made when grasping objects presented using forceinput/vibrotactile output (FV) combined with forcefeedback. The multimodal feedback presented via the prototype is thought to offer considerable potential to supporting skilled and semi-skilled workers to perform remote tasks involving fine motor control.
\end{abstract}

\section{Keywords}

Haptic; Tactile; Tangible, Teleoperation; Vibrotactile

\section{ACM Classification Keywords}

H.5.2. User Interfaces: Haptic I/O

\section{Introduction}

While teleoperation has most commonly been used to support medical, space and defense initiatives, it can also be applied to tasks where skilled or semi-skilled labor is needed (e.g. remote factory work). This would assist organizations who may experience difficulties recruiting due to their location. Haptic-based 


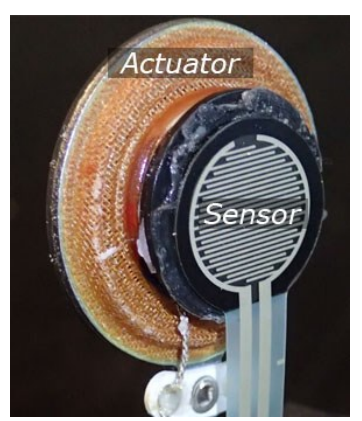

Figure 2: FV Module
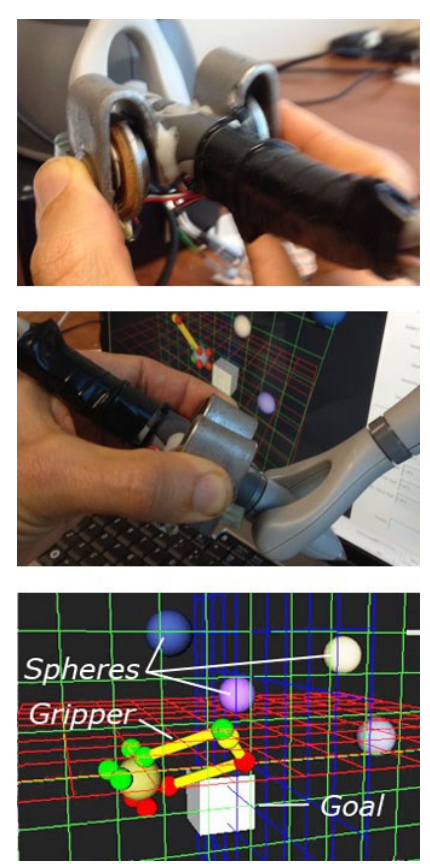

Figure 3: Prototype and Virtual Environment telepresence could be used for a wide variety of more routine work such as factory and farm labor if the interface could be both low-cost and capable of providing the depth of sensation to support generalized interaction for modestly trained individuals. It is not enough for a user to know that they have collided with an object and push it around. They must be able to grasp an object, apprehend its form and surface characteristics, and then manipulate it quickly and without few or no errors. In short, the interaction must become more natural and tangible.

This paper describes a first step towards determining ways to use touch to support manual tasks, through the development of a low-cost sensor/actuator that measures force and provides vibrotactile feedback (termed: FV). A prototype has been developed, enabling users to perform 'picking and placing' tasks. These types of tasks are often performed within a factory environment. The long term goal of our research is to identify the potential of using such a solution in a telecollaborative context.

\section{Related Work}

Force-feedback systems are known to be expensive to purchase. However, lower cost technologies such as the Geomagic Touch (www.geomagic.com) and Novint Falcon (www.novint.com) are available for integration with systems. The user can grasp with the end effector (stylus or ball-like grip) to interact with a virtual or remote surface while feedback is presented to his/her hand.

Extending a low-cost platform, such as the Geomagic Touch, to accommodate more sophisticated interactions such as grasping has been tried several times. Barbagli
[1] added four additional channels of force feedback to a three-degree-of-freedom haptic input/output device to support multi-finger, multi-hand exploration and manipulation of virtual objects. Debus et al. [3] developed vibrotactile transducers to provide more tangible sensations to enhance force feedback. However in these cases, additional capability was provided at considerable complexity and expense.

\section{Prototype Designs}

Bianchi et al. [2] stated that "research on tangible interaction and digital haptics has rarely intertwined". In our literature search, we found only a few similar examples of FV additions to haptic systems. Stuart et al. [11] examined multimodal (combinations of video, audio and vibrotactile) feedback in conjunction with pressure sensing when applied to handheld devices. They found that selection using auditory and tactile feedback could be as rapid as visual only cues, but not as accurate.

Using the research through design methodology [12], we worked through numerous FV prototypes that would sense force and provide tangible user feedback that could be combined easily with other systems (Figure 1). These efforts produced a simple transducer that had two low-cost components - an acoustic-quality actuator coupled with a resistive force sensor (Figure 2). A pressing force applied by the user can be used to move one axis of the end effector, such as a finger. Contact by the end effector results in reality-based waveforms being sent to the actuator, as suggested by Okamoto et al. [8] and Okamura et al. [9]. This allows the user to develop a sense of what the surface of a remote object feels like. 
Table 1: Errors by Condition

\begin{tabular}{|c|c|}
\hline Modality & Errors \\
\hline $\begin{array}{c}\text { Visual with Vibrotactile } \\
\text { output }\end{array}$ & 19 \\
\hline Visual Only & 14 \\
\hline $\begin{array}{c}\text { Visual with Force Feedback } \\
\text { Visual with Force Feedback } \\
\text { \& Vibrotactile }\end{array}$ & 7 \\
\hline
\end{tabular}

Table 2: Mean Task Completion Times

\begin{tabular}{|c|c|c|}
\hline Alias & Session 1 & Session 2 \\
\hline P01 & 63.57 & 57.22 \\
\hline P02 & 73.03 & 81.28 \\
\hline P03 & 55.94 & 52.38 \\
\hline P05 & 41.30 & 30.68 \\
\hline P06 & 67.56 & 57.15 \\
\hline P08 & 42.74 & 35.88 \\
\hline P10 & 43.70 & 31.07 \\
\hline Average & 55.41 & 49.38 \\
\hline
\end{tabular}

The prototype described in this paper was developed to explore the relationship between traditional haptics enhanced by force sensing and vibrotactile feedback. A Geomagic Touch was selected for use in the study. Two FV modules were attached to the device, near the base of the stylus (Figure 3 ). These modules support a pincer-like gripping action, enabling the user to better grasp a virtual object.

Mounted directly underneath the sensors are vibrotactile actuators that are associated with the sensor. Using a Phidgets interface kit

(www.phidgets.com) and the Microsoft XAudio2 API, up to eight sensors and their associated vibrotactile elements can be operated.

\section{Experimental Design and Procedure}

'Picking and placing', such as positioning screws in an 'engine block', is a common factory task. Such an activity is relatively straightforward for individuals who exhibit superior levels of manual dexterity and coordination, but can be difficult for automated systems to perform. 'Picking and placing' demands few errors, as these can impact speed and efficiency.

In our study, participants were presented with a 'picking and placing' task where they were asked to interact with a virtual environment to grasp 5 randomly positioned spheres and move each of the spheres into a 'goal'. Contact with each sphere was mapped to a specific vibrotactile frequency with amplitude in proportion to the simulated contact force between the sphere and the gripper element. This was in accordance with Murray et al.'s findings [8]. The left and right gripper interface elements had their own vibrotactile emitters. Force applied to the pressure sensors on the
Geomagic Touch (as shown in Figure 2) would cause the grippers to close. Relaxing pressure on the sensors would cause the grippers to open.

The following conditions were examined in our study:

- Visual only

- Visual with force feedback

- Visual with vibrotactile output

- Visual with force feedback and vibrotactile output

Ten participants ( 2 women, 8 men, aged 21 - 54) were recruited for the exploratory study. All participants were information workers and comfortable with technology. None had prior experience with tactile or haptic systems other than videogame controllers and mobile phones.

Participants were introduced to the prototype, and given as much time as they needed to become comfortable with using the device to grasp virtual objects. The level of vibrotactile amplitude was adjusted during this time to user preference. All participants were instructed to complete the task of placing all spheres in the goal as quickly as possible. In the virtual environment, it was possible to knock the spheres out of range. Any sphere that was not placed in the goal was counted as an error. Time for each task was calculated from the moment that the Start/Next button was pressed to the time that the last sphere was placed in the goal. If the participant was unable to place all the spheres in the goal, the timer was arbitrarily stopped, and the next session begun. 


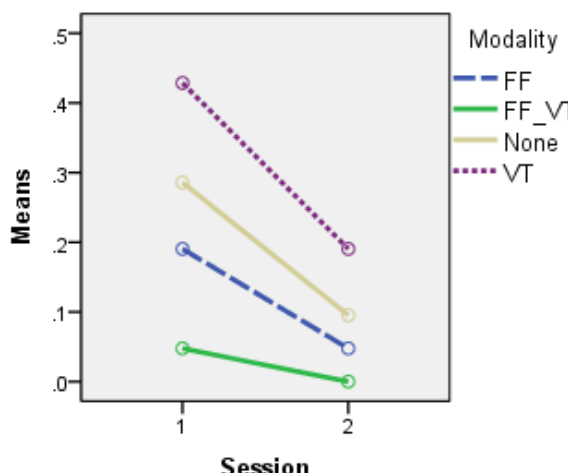

Figure 4: Error Count Means by Session

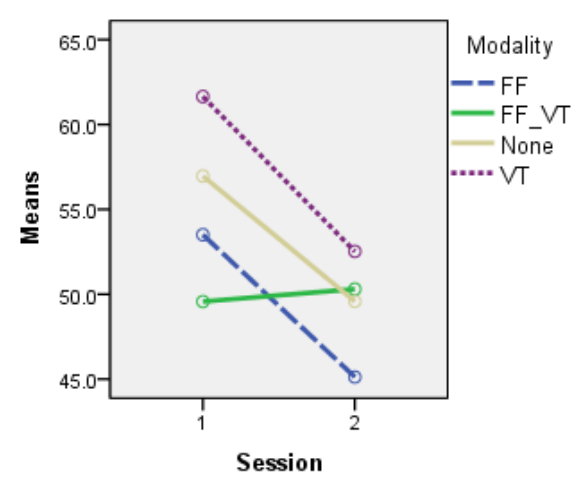

Figure 5: Task Time Means by Session
Errors were calculated per task by subtracting the number of successfully positioned spheres in the goal from the total number of spheres presented in the task.

\section{Results and Discussion}

Participants adapted quickly to the interface. Average completion time for all participants was $55.4 \mathrm{~s}$ per task (SD: $25.0 \mathrm{~s}$ ) for the first session and 49.4s (SD: 22.8s) for the second session. A repeated measures ANOVA was conducted. However, no significant effect could be detected by completion time $(F(1,20)=3.617$, $\mathrm{p}=0.072$ ).

\section{Errors}

Seven participants committed a total of 44 errors.

These included pushing the sphere beyond the range of the gripper while attempting to grasp it, and placing the sphere in a location where it was occluded by the goal.

The majority of errors occurred in the first session (37 vs. 7) (Table 1). Examining the means plots presented in Figure 4, participants appeared to make fewer errors when the combination of the force-feedback and vibrotactile modalities were used to perform the tasks.

$44.1 \%$ of errors were made in the visual with vibrotactile output condition. In other studies examining vibrotactile interaction including Debus et al. [3] and Foehrenbach et al. [5], presenting vibrotactile feedback by itself did not appear to provide a significant reduction in errors when performing tasks. On the other hand, findings from our study suggest that fewer errors are made when vibrotactile feedback and force-feedback are presented together, compared to vibrotactile output alone. However, further study would be needed to confirm this.
Task Time

Prior to each session, participants were asked to place the spheres in the goal as quickly and accurately as possible. Completion times are presented in Table 2. Findings have suggested that participants were generally faster in their second session than their first session, and that the times to complete the second task were more consistent.

Looking at the task time means across the sessions in Figure 5, the results were not as clear as with the error count (Figure 4). For session 1, the relationship between the modalities reflected the pattern seen in the error means for the first session. The order in time taken per task was initially the same as the ordering for errors committed. In other words, the vibrotactile modality took the most time (61.6s), and the force feedback plus vibrotactile modality took the least (49.6s). However, when the users performed the tasks the second time, this pattern changed. The forcefeedback plus vibrotactile took longer ( $50.3 \mathrm{~s}$ vs $49.5 \mathrm{~s}$ ) and the spread of times increased as well (SD: $18.5 \mathrm{~s}$ vs $28.5 \mathrm{~s}$ ). For all other cases, the average time for the other conditions decreased as training improved. The decrease in performance for the force feedback plus vibrotactile modality was a perplexing result, and may reflect the small sample size. However, Massimino identified a similar effect when he was combining modalities to support users when performing a peg-inhole task using the E2 Master-Slave manipulator. In this case, the addition of vibrotactile to a force-feedback plus audio configuration increased completion times for the task [6]. 


\section{Conclusions and Future Work}

The goal of this exploratory study was to identify the impact of modality on picking and placing tasks.

Findings indicated that there may not be a need for an additional costly force-feedback axis. Rather, a forcesensing/vibrotactile output component mounted to a force feedback base could potentially offer assistance when performing a task. The combination of tangible interaction with force-feedback may provide a low-cost but potentially effective way to enhance simple haptic systems. This study suggests that such a system has the capability to reduce errors made when grasping and releasing, and could possibly be useful for decreasing task time when undertaking a task that requires fine motor skills. Further study would need to be conducted to determine if this is truly the case.

Adding force-sensing components to other devices where the range of "virtual" motion is suitably constrained may be worth pursuing. For example, components can be embedded within in the casing surrounding a smartphone or a videogame controller (e.g. a joystick). As long as the moving base is tracked and used to determine the position and orientation of the gripping "effector", the result may be sufficient. Improvements in the amount and type of vibrotactile information presented may provide a more effective interface (e.g. as observed in the studies by [4] and [10]).

The hardware and software for this study is designed to be extended to support further experiments in low-cost teleoperation. To this end, we intend to configure another Geomagic Touch with the same FV platform and work on connecting it to a manufacturing robot such as Baxter. As such, the next steps will be to develop additional software that supports communication with the target hardware, and the development of software that will support digitizing and virtualizing the work environment so that the operator can operate with responsive haptics without having to account for the types of time lags that are common in teleoperation. Further studies will then focus on developing an effective local human-distant robot interaction with respect to manufacturing-style tasks.

\section{References}

[1] Barbagli, F., Salisbury, K., Devengenzo, and R. Enabling multi-finger, multi-hand virtualized grasping. Robotics and Automation, 2003. Proceedings. ICRA'03. IEEE International Conference on. Vol. 1. IEEE, 2003.

[2] Bianchi, A., et al. Haptics for tangible interaction: a vibro-tactile prototype. Proceedings of the fifth international conference on Tangible, embedded, and embodied interaction. ACM, 2011.

[3] Debus, T., et al. Multichannel vibrotactile display for sensory substitution during teleoperation. Proceedings of SPIE-The International Society for Optical

Engineering. Vol. 4570. 2001.

[4] DiFilippo, D. and Pai, D. K., Contact interaction with integrated audio and haptics. Proceedings of the International Conference on Auditory Display (ICAD). 2000.

[5] Foehrenbach, S., et al. Natural interaction with hand gestures and tactile feedback for large, high-res displays. Bibliothek der Universität Konstanz, 2008.

[6] Massimino, M., Sensory substitution for force feedback in space teleoperation. Diss. Massachusetts Institute of Technology, 1992.

[7] Murray, A., Klatzky, R., and Khosla, P., Psychophysical characterization and testbed validation of a wearable vibrotactile glove for telemanipulation. Presence: Teleoperators and Virtual Environments 12.2 (2003): 156-182. 
[8] Okamoto, S., Wiertlewski, W., and Hayward, V. Anticipatory vibrotactile cueing facilitates grip force adjustment. World Haptics Conference (WHC), 2013. IEEE, 2013

[9] Okamura, A.M., Cutkosky, M.R. and Dennerlein, J.T. Reality-based models for vibration feedback in virtual environments. Mechatronics, IEEE/ASME Transactions on 6.3 (2001): 245-252.

[10] Richmond, J.L. and Pai, D.K., Active measurement of contact sounds. Robotics and Automation, 2000.
Proceedings. ICRA'00. IEEE International Conference on. Vol. 3. IEEE, 2000.

[11] Stewart, C., et al. Characteristics of pressure-based input for mobile devices." Proceedings of the SIGCHI Conference on Human Factors in Computing Systems. ACM, 2010.

[12] Zimmerman, J., Forlizzi, J., and Evenson, S.

Research through design as a method for interaction design research in $\mathrm{HCI}$. Proceedings of the SIGCHI

conference on Human factors in computing systems. ACM, 2007. 